\title{
The effect of surgical gastric plication on obesity and diabetes mellitus type 2: a systematic review and meta-analysis
}

\author{
Hauke Heinrich Georg Meyer, Romualdas Riauka, Zilvinas Dambrauskas, Antanas Mickevicius \\ Department of Surgery, Medical Academy, Lithuanian University of Health Sciences, Kaunas, Lithuania
}

Videosurgery Miniinv 2021; 16 (1): 10-18

DOI: https://doi.org/10.5114/wiitm.2020.97424

\begin{abstract}
Introduction: All the bariatric procedures have evolved greatly over the past decades and laparoscopic greater curvature plication (LGCP) is one of the quite recently introduced techniques lacking systematic evaluation.

Aim: To compare and summarize the current data in the literature in regard to the effect of gastric plication on obesity and diabetes mellitus type 2.

Material and methods: The systematic review and meta-analysis was performed according to the PRISMA guidelines and registered at PROSPERO under the registration number CRD42018114314. The literature in English and German was searched using the MEDLINE (PubMed) and BJS databases for studies published in the last 10 years. A meta-analysis was performed focusing on the effects of this operation on weight loss, glycemia control and improvement of comorbidities.

Results: Mean preoperative body mass index (BMI) ranged from 34.42 to $46.3 \mathrm{~kg} / \mathrm{m}^{2}$. Most of the patients were female. The operation time was in the range from 50 to $192.23 \mathrm{~min}$. Mean follow-up was from one month to 12 years, with most studies having a follow-up of less than 2 years. The postoperative BMI ranged from 28.59 to 38, with reported excess weight loss (EWL\%) in the range 20-70\%. Glycated hemoglobin $\left(\mathrm{HbA}_{1} \mathrm{~d}\right.$ values decreased by up to $5.1 \%$ after surgery, ranging from $5.1 \%$ to $7.5 \%$.

Conclusions: Despite the quality of most of the included studies being low, the present meta-analysis revealed that, in the short term, gastric plication is an effective measure for weight loss, while the effect on diabetes mellitus type 2 is not statistically significant.
\end{abstract}

Key words: bariatric surgery, gastric plication, weight loss, type 2 diabetes.

\section{Introduction}

Overweight and obesity is a growing problem worldwide. Since 1975 obesity prevalence has almost tripled [1]. In 2016, 1.9 billion adults ( $>18$ years old) were overweight, with 650 million being obese (body mass index - BMI $\geq 30 \mathrm{~kg} / \mathrm{m}^{2}$ ). Overall, that equates to $13 \%$ of the population of the world being obese in 2016 [2]. Alongside obesity, diabetes type 2 is also increasing in prevalence, with an estimated 422 million adults suffering from diabetes in 2014, which is around $8 \%$ of the world's adult population. Overweight and diabetes are managed with multiple conservative as well as surgical procedures, where surgical procedures showed a clearly better result in terms of reduction of body fat and a decrease in comorbidities [3-6]. As of now, despite invasiveness and some challenges during the perioperative period, bariatric surgery is the only solution for sustained weight loss and decreasing comorbidities in the morbidly obese [7, 8]. All the bariatric procedures evolved greatly over the past decades, ranging from

\section{Address for correspondence}

Zilvinas Dambrauskas, Department of Surgery, Lithuanian University of Health Sciences, 2 Eiveniu St, 50009 Kaunas, Lithuania,

phone: +370-37-326751, fax:+370-37-327163, e-mail: zilvinas.dambrauskas@lsmuni.lt 
jejunoileal bypass related procedures over Rouxen-Y bypass to restrictive procedures such as sleeve gastrectomy. A rather new approach in bariatric surgery is the laparoscopic greater curvature plication (LGCP) [9]. Gastric plication was first described by Tretbar et al. [10] in 1976 and was initially done in an open way. The laparoscopic approach was proposed by Talebpour et al. [11] and is gaining growing interest as an alternative to other bariatric procedures. New studies have shown that LGCP has a low postoperative complications rate, improves comorbidities of the patients and has the advantage of being less invasive [12]. Laparoscopic greater curvature plication is a bariatric procedure which reduces the stomach volume in a way that the greater curvature of the stomach is folded into itself and fixed with one or multiple rows of stitches, resulting in a restrictive effect [13]. For now, there is no standardized technique for LGCP. However, there are 2 main steps for the operation. Firstly the greater curvature of the stomach has to be mobilized, followed by a plication (suturing) of the gastric fold [14-18]. Fried et al. and Gudaityte et al. $[17,19]$ found no significant differences between single-row plications compared to two-row plications for the effectiveness and safety of the procedure. The literature is still controversial as to which type of suture is better, with some studies reporting two rows of running sutures [14], others two rows of interrupted sutures $[15,16,20]$ and some reporting a combination of both running and interrupted sutures [18].

\section{Aim}

The aim of this study was to compare and summarize the current literature in regard to the outcomes of gastric plication in obesity and diabetes mellitus type 2: to assess the short-term and longterm weight loss, the changes in glycated hemoglobin $\left(\mathrm{HbA}_{1 \mathrm{c}}\right)$ and necessity of antidiabetic medica-

Table I. Search terms

"Gastric plication", "Gastric plicature", "Great curvature plication", "Gastric vertical plication", "Laparoscopic gastric greater curvature plication", "Laparoscopic gastric plication", "diabetes mellitus", "diabetes", "diabetes surgical treatment”, "obesity", "surgical treatment for obesity",

"type 2 diabetes mellitus", "bariatric surgery", "morbid obesity"

Languages of searched articles were restricted to English and German tions and/or insulin therapy after gastric plication procedure; and to investigate the possible improvement of comorbidities.

\section{Material and methods}

The systematic review was performed according to the PRISMA [21, 22] guidelines and registered at PROSPERO [23] under the registration number CRD42018114314.

\section{Systematic search strategy}

The literature was searched and reviewed systematically using the MEDLINE (PubMed) and BJS databases for studies published in the last 10 years (from 2010 to 2018). Various text words and medical subject heading (MeSH) terms were selected (Table I).

\section{Eligibility}

Studies which reported the long- or short-term effect of gastric plication surgery on objectively measured weight loss were included; changes in the pharmaceutical treatment of diabetes mellitus after gastric plication, changes in insulin secretion and need of additional insulin therapy after gastric plication were investigated. Studies published earlier than 10 years ago, studies focusing on other bariatric procedures and animal studies were excluded. Abstracts, unpublished studies, guidelines, newspaper articles, small case reports and studies where the full text was unavailable were also excluded.

\section{Study selection}

From November 2017 to December 2018, the databases were searched using the keywords mentioned in Table I. Terms were combined using the words "and" and "or". The author screened the titles for eligibility. First, the abstracts were screened for relevance; seemingly relevant abstracts were screened in the full text. Disagreements were solved by consensus and discussion. In the case of duplicated publications, the most complete and recent studies were selected. The primary and secondary outcomes were extracted from the full texts.

\section{Methodological quality}

The authors assessed the bias risk and quality of included studies, by considering the following characteristics: completeness of outcome data, specifici- 


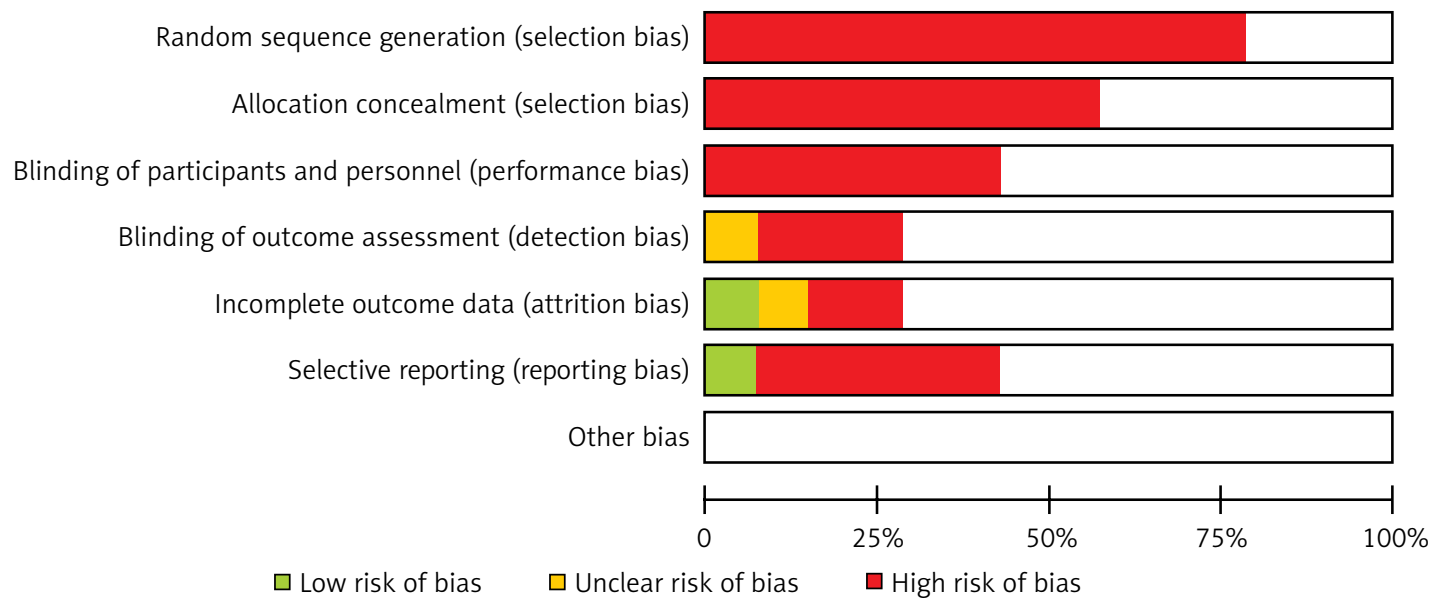

Figure 1. Risk of bias of the included studies

ty of studied intervention, missing patient characteristics and other sources of bias (Figure 1).

\section{Outcome measures}

The primary outcomes, objective weight loss and changes in the therapy of diabetes mellitus type 2 (DM T2) were extracted from the included articles. Data on the number of patients who underwent gastric plication and follow-up weight loss as well as changes in the treatment of diabetes were obtained.

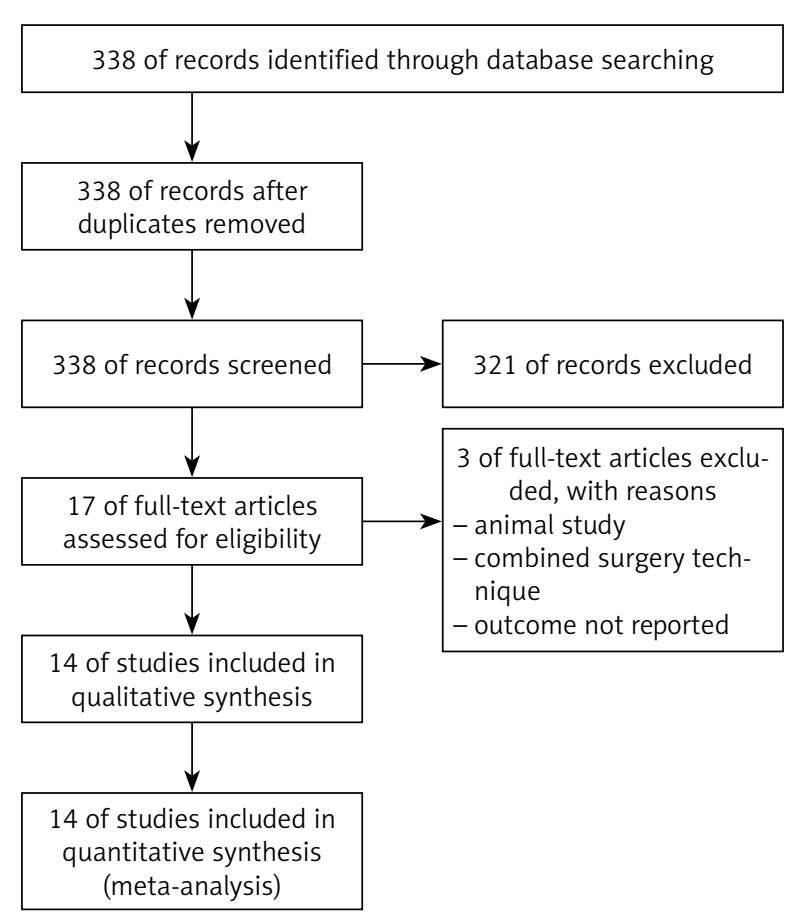

Figure 2. PRISMA diagram showing the selection of articles for review
Secondary outcomes were the time frame of effect and complications after surgery.

\section{Quality appraisal}

The selected studies were appraised according to the PRISMA statement. Incomparable groups, information bias because of incomplete or inadequate outcome measures and selection bias by incomplete follow-up were controlled. The final decision regarding the inclusion of the articles was reached in a meeting; discrepancies were solved in a consensus manner.

\section{Statistical analysis}

A random effect model was used to pool the different outcomes of the studies in order to show an overall outcome of weight loss and change in diabetes treatment. To estimate heterogeneity between the included studies, tau values in the forest plots were used. All statistical analysis was performed using RevMan version 5.3.

\section{Results}

Using the above-named search terms, 338 publications were found. After exclusion of 321 articles, the remaining 17 articles were chosen for further reading. Three articles did not match the criteria for critical appraisal. The remaining 14 articles were read in full text and extracted for data (Figure 2).

The 14 studies included 2071 patients. Most articles varied strongly in sample sizes, ranging from 13 to 800 patients. Most of the studies included women and men, while one study included women only [24]. All of the remaining 13 studies had a majority of female patients. Five studies only investigat- 
ed the effect of gastric plication on weight loss and 1 study reported only outcomes on diabetes mellitus type 2. All studies had participants with a mean preoperative body mass index (BMI) of $>35 \mathrm{~kg} / \mathrm{m}^{2}$, with only one study from China reporting a mean preoperative BMI of $34.42 \mathrm{~kg} / \mathrm{m}^{2}$. Seven studies had a follow-up of 1 year or less, two studies reported a follow-up to 2 years and five studies presented data of a follow-up period of over 2 years. Only 2 studies reported a follow-up of over 5 years. Time of surgery varied over the studies, from around $50 \mathrm{~min}$ [16] to $192.23 \min [20]$.

The outcome measurement differed among the included studies, some using preoperative and postoperative BMI, others excess body mass index loss (EBMIL), total weight loss (TWL) or others for weight loss, and $\mathrm{HbA}_{1 \mathrm{c}}$ changes, withdrawal or decrease in oral antidiabetic medications and/or insulin for diabetes mellitus type 2 . Six studies reported the effect by means of pre- and postoperative $\mathrm{HbA}_{1 c}$ values. The remaining 3 studies reported the outcome as the percentage of patients achieving either remission or improvement of diabetes mellitus type 2 by means of reduced or withdrawn medications. Only 4 studies compared the BMI pre- and postoperatively, indicating the standard deviation and only 4 mentioned the standard deviations in regard to $\mathrm{HbA}_{1 \mathrm{c}}$ values. Other studies did not report standard deviations, used different units to display the weight loss and effect on diabetes mellitus type 2, or did not report one of the outcomes.
The analysis was performed for those studies where the standard deviations were reported and comparable outcome data were provided and where more than two studies were available for the time outcome.

The outcomes of weight loss and improvement or remission of diabetes mellitus type 2 are presented in forest plots (Figures 3-9). The smallest postoperative weight loss was a loss of 2.9 BMI after 1-month follow-up [24]. The largest weight loss was $9.93 \mathrm{~kg} / \mathrm{m}^{2}$ loss of BMI postoperatively $(95 \% \mathrm{Cl})$.

An overall test for heterogeneity between the included studies was performed in each forest plot and gave consistently significant results ( $I^{2}$ values in Figures 3-9).

In most of the included studies the preoperative BMI was in a lower range, with only one study having a $\mathrm{BMI}>45 \mathrm{~kg} / \mathrm{m}^{2}$. Of importance is that there might be a correlation between a higher preoperative BMI and weight loss, with one study pointing out that the total EWL was higher in a group with a preoperative $\mathrm{BMI}$ of $<45 \mathrm{~kg} / \mathrm{m}^{2}$ when compared to a group who had a BMI of $>45 \mathrm{~kg} / \mathrm{m}^{2}$ [15].

In a recent study Gudaityte et al. [19] investigated the intermediate results of LGCP in 61 patients with a mean preoperative BMI of $46.4 \mathrm{~kg} / \mathrm{m}^{2}$. In their 3-year follow-up, they concluded that LGCP has only a moderate effect on weight loss, with an EBMIL\% of $47.25 \%, 44.8 \%, 41.9 \%$ at 1,2 and 3 years, respectively. They also stated that of the 19 patients having diabetes preoperatively, the $\mathrm{HbA}_{1 c}$ values de-

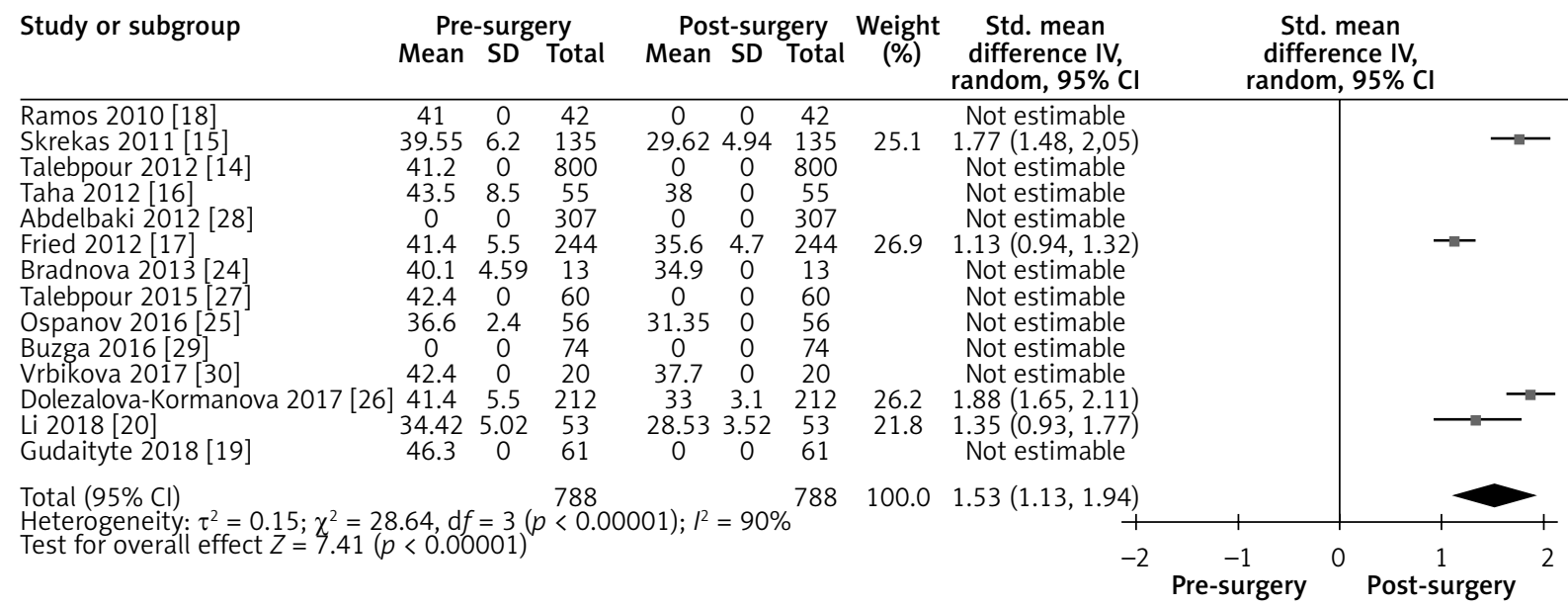

Figure 3. Influence of LGCP on BMI: Random effect forest plot illustrating the total effect of LGCP on weight loss. Weight is put in values as preoperative and postoperative BMI where available. Only four studies illustrated the pre- and postoperative BMI values with standard deviations. The other studies are listed, but not included in the statistical analysis. The $l^{2}$ value shows a high heterogeneity between the studies 


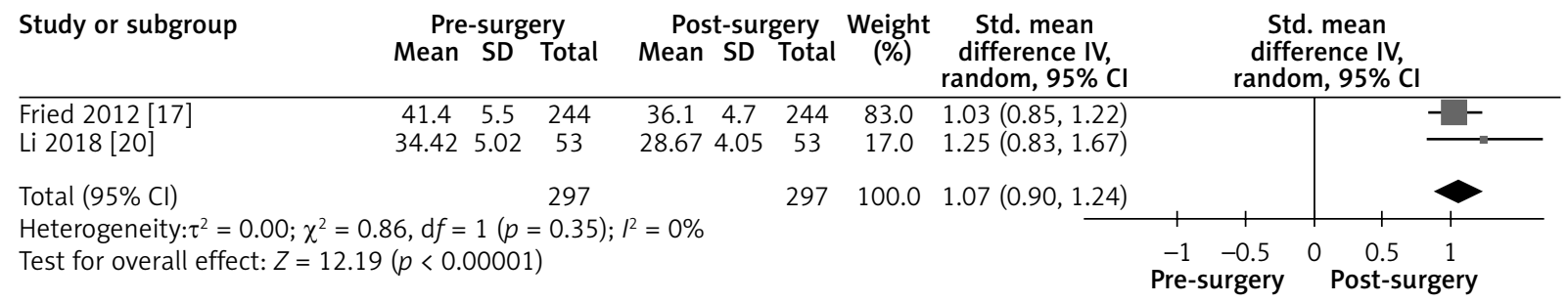

Figure 4. Effect of LGCP on BMI after 6 months: Random effect forest plot indicating the effectiveness of LGCP in terms of BMI change after the surgery at 6 months. Only two studies illustrated the 6 months outcome on weight loss reporting BMI values with standard deviation. Other studies were not included to reduce heterogeneity

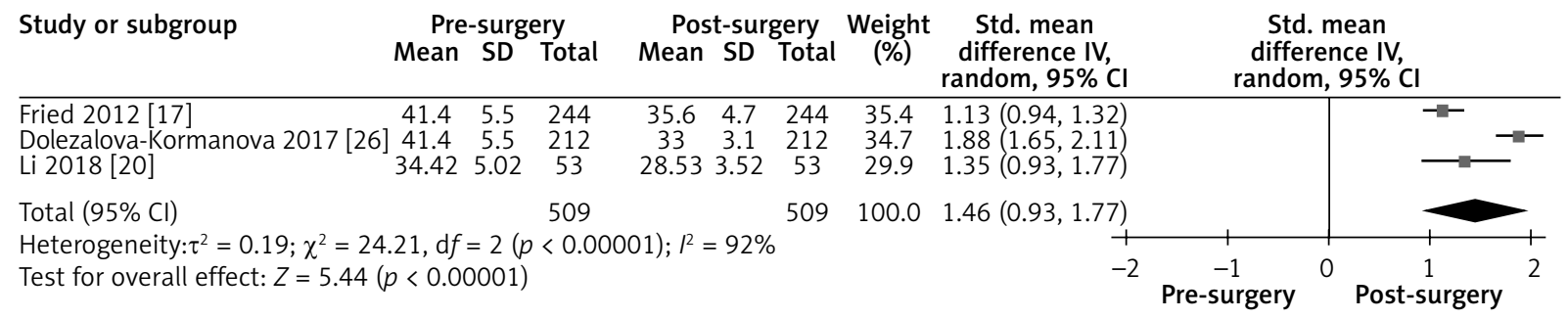

Figure 5. Effect of LGCP on BMI after 12 months: Random effect forest plot representing the effectiveness of weight loss after LGCP at 12 months postoperatively. One additional study reported the BMI with standard deviations after surgery. Heterogeneity between the three included studies is high $\left(I^{2}=92 \%\right)$

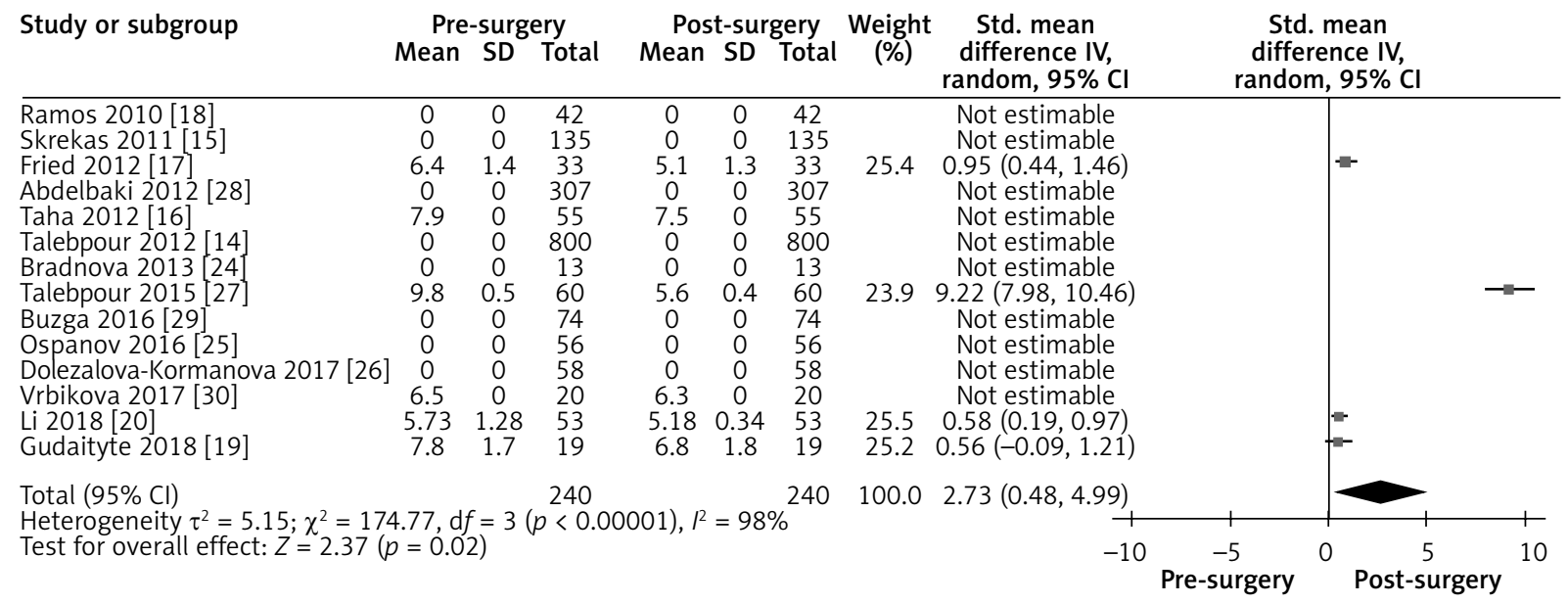

Figure 6. Influence of LGCP on diabetes mellitus: random effect forest plot showing the effect of LGCP on $\mathrm{HbA}_{1 \mathrm{c}}$ values after surgery. Only the studies representing data with their standard deviation were included in the statistical analysis. $I^{2}$ value shows a high heterogeneity between the studies

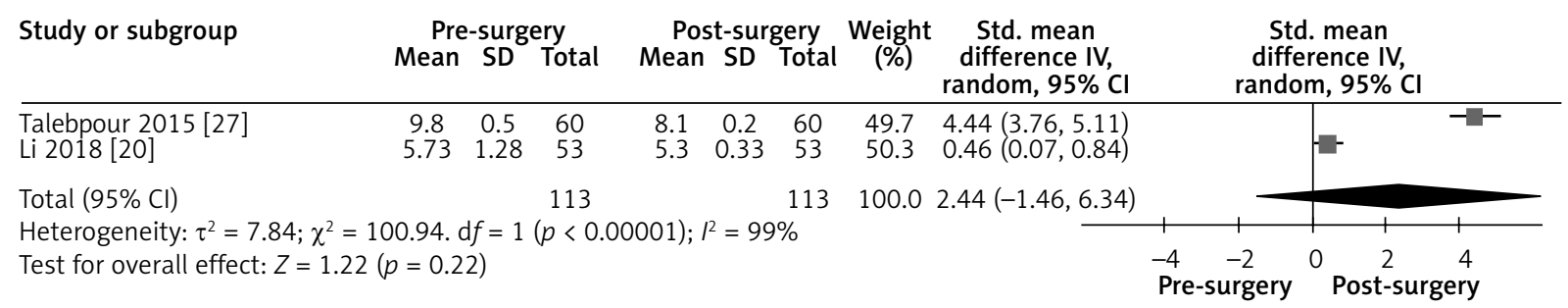

Figure 7. Effect of LGCP on $\mathrm{HbA}_{1 \mathrm{c}}$ after 3 months: Random effect forest plot summarizing the effect of LGCP on $\mathrm{HbA}_{1 c}$ at 3 months after surgery. There is very high heterogeneity between the studies $\left(I^{2}=99 \%\right)$. These results are also not statistically significant, as the $95 \% \mathrm{Cl}$ includes the null value 


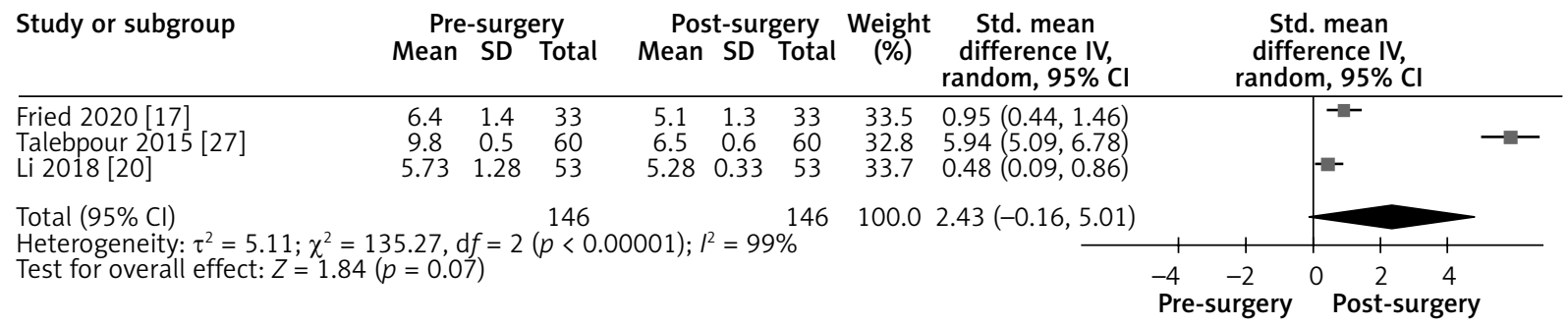

Figure 8. Effect of LGCP on HbA1c after 6 months: Random effect forest plot summarizing the effect of LGCP on $\mathrm{HbA}_{1 c}$ at 6 months after surgery. There is very high heterogeneity between the studies $\left(I^{2}=99 \%\right)$. These results are also not statistically significant, as the $95 \% \mathrm{Cl}$ includes the null value

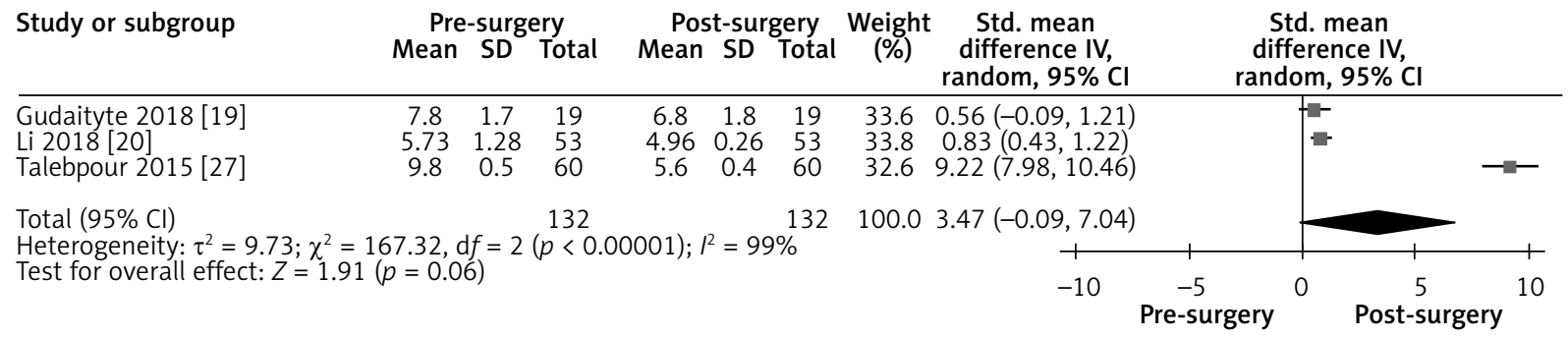

Figure 9. Effect of LGCP on $\mathrm{HbA}_{1 c}$ after 12 months: Random effect forest plot summarizing the effect of LGCP on $\mathrm{HbA}_{1 \mathrm{c}}$ at 12 months after surgery. There is very high heterogeneity between the studies $\left(I^{2}=99 \%\right)$.

These results are also not statistically significant, as the $95 \% \mathrm{Cl}$ includes the null value

creased from 7.8 to 6.8 after 3 years $(p=0.001)$. In this sample size, $72.3 \%$ of the preoperative diabetics achieved either remission or improvement after the surgery, with $27 \%$ of patients undergoing the procedure achieving DM T2 remission. Other comorbidities also improved after the surgery, with $38.3 \%$ achieving remission and $29.8 \%$ showing an improvement in hypertension.

A study by Ospanov et al. [25] assessed the connection between gastric greater curvature plication combined with the Nissen fundoplication effect on obesity and other comorbidities such as diabetes mellitus, hypercholesterolemia, and gastroesophageal reflux. Apart from the clear effect on weight loss, a significant amount of patients was found with remission and/or improvement of type 2 diabetes mellitus ( $p=0.03$ ), hypercholesterolemia ( $p=$ 0.0001 ) and gastroesophageal reflux (GERD) subjective symptoms, caused by esophagitis ( $p=0.0001)$.

While most studies prove LGCP as safe and feasible in the short-term treatment of obese people with and without DM type 2 [14-19, 24-28], other studies point out the risks of weight regain threatening the procedure to be unsustainable and inferior in the longer term $[14,16,28]$. The main risk factor for weight regain is dependent on the patients' lifestyle after the operation [14].
The study of Buzga et al., with 12-month follow-up on DM T2, reported LGCP to be effective for DM T2 improvement, with $33 \%$ of patients discontinuing oral hypoglycemic medication and insulin and $88 \%$ reaching a target $\mathrm{HbA}_{1 \mathrm{c}}$ of $<4.8 \%$; however, the author pointed out that the sample size of patients was insufficient to make a definitive statement about the effectiveness of LGCP [29].

Controversially, another study by Taha [16] reported the $\mathrm{HbA}_{1 \mathrm{c}}$ levels of $7.5 \%$ postoperatively compared to $7.9 \%$ preoperatively, in 55 patients at 12 months, with no patients withdrawing their hypoglycemic medications.

The study from Egypt [16] determined an EWL of $35 \%$ after 12 months, with $\mathrm{BMI}$ decreasing to 38 from 43.5 preoperatively. However, the trend of insufficient weight loss and weight regain after intermediate and long-term follow-up was found here already after 6 months, with $23 \%$ of patients ceasing to lose weight at 6 months, and $11 \%$ regaining weight after 9 months.

Two small studies [24, 30], focusing on the effect on diabetes mellitus type 2 after LGCP, estimated an improvement in 6-month follow-up not only in DM type 2 but also in the metabolic profile of the patients. In both studies, GIP levels increased after the plication, with Vrbikova et al. [30] reporting a de- 
crease of C peptide as a marker of DM T2 after LGCP and Bradnova [24] finding a statistically significant decrease in $\mathrm{HbA}_{1 c}(p<0.0001)$ and improvement of hyperglycemia $(p<0.05)$. DM T2 was resolved in $4 / 20(20 \%)$ patients and improved in $12 / 20(60 \%)$ at 6-month follow-up. Total insulin secretion did not change significantly $(p<0.001)$. Both studies reported an effective weight loss after the procedure.

The Dolezalova-Kormanova et al. study [26] tried to determine the difference of effects by determining cluster groups according to age and BMI preoperatively. The most successful weight loss was in a cluster of young females ( $<50$ years old), with a mean BMI of $38.5 \mathrm{~kg} / \mathrm{m}^{2}$. The study estimated the probability of success after fat percentage preoperatively had a chance of success of $90 \%$, a person with $60 \%$ had only a $50 \%$ chance of success after LGCP $(p<0.001)$.

The largest study from Fried et al. [17] with 244 patients, but a shorter follow-up of only up to 18 months, found LGCP to be feasible for weight loss and improvement of $\mathrm{DM} \mathrm{T2}$, with $\mathrm{HbA}_{1 \mathrm{c}}$ decreasing to $5.1 \%(p<0.001)$ and EBMIL of $31.4 \%(p<0.001)$. At 6 months, the BMI decreased to 36.1 from $41.4 \mathrm{~kg} / \mathrm{m}^{2}$ preoperatively. Also, $96.9 \%$ of patients that had DM T2 achieved remission or improvement by 6 months after surgery. The authors found a correlation with higher preoperative BMI leading to less weight loss, although by the 9-month follow-up there seemed to be no statistically significant difference in their sample size.

Most studies only had a short-term follow-up period of 1-18 months [16-18, 20, 24, 25, 27, 29, 30], which revealed an effective decrease in weight and improvement or remission in diabetes mellitus over that time. Only 5 studies published longer follow-up data, 4 of which reported an average follow-up to 5 years, and only one study had a follow-up of 10 years.

Doležalova-Kormanova et al. [26] published a study with a follow-up time of 5 years. With a significant BMI decrease for the first 2 years $(p<0.001)$, it proved LGCP to be effective in the short term. A plateau from 3 to 4 years and an increase $(p<0.01)$ in BMI at 5 years postoperatively, however, revealed a less successful outcome in the medium and longer term $(p<0.001)$. Along with other studies focusing on the effect on diabetes mellitus type 2 , the authors indicated LGCP to be feasible and effective in the treatment of diabetes mellitus type 2 , with an improve- ment rate of $65.5 \%$ at 5 years in 57 patients who had diabetes mellitus type 2 before surgery. A shortterm effect on diabetes mellitus type 2 was shown in a reduction in oral hypoglycemic agents and/or insulin intake.

Skrekas et al. [15] followed the weight loss after LGCP for 2 years, observing a marked decrease of EWL, with a loss of $51.7 \%$ at 6 months and $67.1 \%$ at 12 months.

Ramos et al. [18] found a similar effect in the short term, reaching a EWL up to $62 \%$ at 18 months. However, that the decrease became less marked in longer follow-up (65.1\% at 24 months). This is in accordance with other long-term studies [14, 26]. They also found that with higher preoperative BMI, the inadequate weight loss was doubled when compared to a control group with a BMI under $45(p \leq 0.001)$. The effectiveness of the procedure was not affected by single (21.4\% insufficient weight loss) or multiple plications (21.5\% insufficient weight loss).

The study from China by Li et al. [20] reported that a less prominent effect on weight loss after LGCP as well as no significant difference in $\mathrm{HbA}_{1 c}$ and other blood sugar profiles was found pre- and postoperatively.

Talebpour, who introduced the laparoscopic gastric plication, published multiple studies on this procedure [14, 27]. In his 2012 [14] study, the biggest one to date, he found LGCP to be effective in shortterm treatment of obesity. He achieved an EWL loss of $70 \%$ after 2 years, $55 \%$ after 5 years. The authors pointed out that there is a tendency of weight regain after 4 and 5 years after the surgery. This tendency was proved by weight regain after LGCP in the longer term, with $5.5 \%$ of patients experiencing an increase in weight after 4 years, and a whole $31 \%$ after 12 years. In conclusion, Talebpour et al. [14] reported acceptable results as a method to treat morbid obesity. Complication rates are low $[14,16,17,19$, 26], while the costs are significantly lower for LGCP than for other types of bariatric surgery [14, 15]. All studies determined that the side effects were mostly nausea and vomiting, with the complications and failure of the procedure being related to the preoperative $\mathrm{BMI}(p=0.063)$ [15].

In a later, short-term follow-up study from Talebpour et al. [27], LGCP was not only found effective in the short term for obesity, with a significant weight loss after 6 months, but also for other comorbidities. All of the 60 included patients suffering from 
DM T2 achieved either remission (92\%) or improvement (8\%). There was a marked decrease in blood pressure $(p=0.001), \mathrm{HbA}_{1 c}(p=0.002)$, weight $(p=$ $0.001)$, total cholesterol $(p=0.001)$ and triglycerides $(p=0.001)$.

\section{Discussion}

The current data show that LGCP has a promising short-term result for weight loss and that there might be some advantages of gastric plication over other existing bariatric procedures. Potential advantages are the possibility for reversibility, lack of resection, lower invasiveness and lower cost. It should be noted that despite those advantages LGCP cannot eliminate the risk of complications completely. Although the risk and number of complications are low, evidence shows that LGCP is inferior and less sustainable than other bariatric procedures, mainly laparoscopic sleeve gastrectomy [16]. Weight regain started at 6-12 months [16] and was most evident at 4-5 years postoperatively [14].

The longer term effect of gastric plication on weight loss is not dependent on the technical details of the surgical procedure, but on the ability of the patient to follow and maintain the lifestyle changes [14]. Intermediate follow-up results show only a modest weight loss, increased hunger being the main risk factor for unsatisfactory weight loss [19].

The remission of diabetes in most studies was based on the $\mathrm{HbA}_{1 \mathrm{c}}$ value decreasing postoperatively (>0.5 decrease), which shows a mean decrease in $\mathrm{HbA}_{1 \mathrm{c}}$ of 1.625 over the 6 studies $[16,17,19,20,27,30]$. Other studies determined DM T2 remission by either withdrawal of medication and/or insulin.

Critical appraisal revealed a lack of information especially on long- and intermediate-term follow-up and a high risk of bias, due to different follow-up times, sample sizes and methods of outcome reporting. There are many limitations to this study because of a lack of high-quality data concerning outcomes of gastric plication as well as patients' perioperative education in the current literature. As known from the different studies, education provided before and after surgery may improve outcomes of other bariatric surgery procedures [31-33]. A lack of short- and long-term follow-up data makes it difficult to predict the true effect of gastric plication. The majority of the included studies have a low number of patients included. Although this study includes 2071 patients, only 5 studies had over 100 patients. Further- more, there is a high need for medium- and longterm follow-ups regarding LGCP, with only 2 studies reporting a follow-up of over 3 years.

Therefore, to make valid estimations, mediumand long-term follow-up studies with larger sample sizes and a standardized method of outcome reporting are needed.

This meta-analysis attempts to provide an overview of the current literature on this novel procedure.

\section{Conclusions}

Though the quality of the included studies is relatively low, the present meta-analysis shows that, in the short term, gastric plication is an effective measure for weight loss and control of obesity-related comorbidities, while the effect on diabetes mellitus type 2 is not statistically significant.

\section{Conflict of interest}

The authors declare no conflict of interest.

\section{References}

1. Hossain P, Kawar B, El Nahas M. Obesity and diabetes in the developing world - a growing challenge. N Engl J Med 2007; 356: 213-5.

2. Obesity and overweight [Internet]. Who.int. 2018 [cited 1 January 2019]. Available from: https://www.who.int/news-room/ fact-sheets/detail/obesity-and-overweight

3. Wojciak P, Pawłuszewicz P, Diemieszczyk I, et al. Laparoscopic sleeve gastrectomy: a study of efficiency in treatment of metabolic syndrome components, comorbidities and influence on certain biochemical markers. Videosurgery Miniinv 2020; 15: 136-47.

4. Wirth A, Wabitsch M, Hauner $H$. The prevention and treatment of obesity. Dtsch Arztebl Int 2014; 111: 705-13.

5. Rubino F, Gagner M. Potential of surgery for curing type 2 diabetes mellitus. Ann Surg 2002; 236: 554-9.

6. Wolfe BM, Kvach E, Eckel RH. Treatment of obesity: weight loss and bariatric surgery. Circ Res 2016; 118: 1844-55.

7. Stefura T, Skomarovska O, Wysocki M, et al. Challenges associated with bariatric surgery - a multi-center report. Videosurgery Miniinv 2019; 14: 526-31.

8. Sjöström L, Lindroos AK, Peltonen $M$, et al. Lifestyle, diabetes, and cardiovascular risk factors 10 years after bariatric surgery. N Engl J Med 2004; 351: 2683-93.

9. Buchwald $\mathrm{H}$. The evolution of metabolic/bariatric surgery. Obes Surg 2014; 24: 1126-35.

10. Tretbar LL, Taylor TL, Sifers EC. Weight reduction. Gastric plication for morbid obesity. J Kans Med Soc 1976; 77: 488-90.

11. Talebpour M, Amoli BS. Laparoscopic total gastric vertical plication in morbid obesity. J Laparoendosc Adv Surg Tech A 2007; 17: 793-8. 
12. Li Y, Wang B, Huang Y, et al. Clinical outcomes of laparoscopic greater curvature plication and laparoscopic sleeve gastrectomy: a case-matched control study. Obes Surg 2019; 29 387-93.

13. Park YH, Kim SM. Short-term outcomes of laparoscopic greater curvature plication and laparoscopic sleeve gastrectomy in patients with a body mass index of 30 to $35 \mathrm{~kg} / \mathrm{m}^{2}$. Yonsei Med J 2017; 58: 1025-30.

14. Talebpour M, Motamedi SM, Talebpour A, Vahidi H. Twelve year experience of laparoscopic gastric plication in morbid obesity: development of the technique and patient outcomes. Ann Surg Innov Res 2012; $6: 7$.

15. Skrekas G, Antiochos K, Stafyla VK. laparoscopic gastric greater curvature plication: results and complications in a series of 135 patients. Obes Surg 2011; 21: 1657-63.

16. Taha O. Efficacy of laparoscopic greater curvature plication for weight loss and type 2 diabetes: 1-year follow-up. Obes Surg 2012; 22: 1629-32.

17. Fried M, Dolezalova K, Buchwald JN, et al. Laparoscopic greater curvature plication (LGCP) for treatment of morbid obesity in a series of 244 patients. Obes Surg 2012; 22: 1298-307.

18. Ramos A, Galvao Neto M, Galvao M, et al. Laparoscopic greater curvature plication: initial results of an alternative restrictive bariatric procedure. Obes Surg 2010; 20: 913-8.

19. Gudaityte R, Adamonis K, Maleckas A. Laparoscopic gastric greater curvature plication: intermediate results and factors associated with failure. Obes Surg 2018; 28: 4087-94.

20. Li YH, Wang BY, Huang YC, et al. Clinical outcomes of laparoscopic greater curvature plication and laparoscopic sleeve gastrectomy: a case-matched control study. Obes Surg 2019; 29: 387-93.

21. Liberati A, Altman D, Tetzlaff J, et al. The PRISMA statement for reporting systematic reviews and meta-analyses of studies that evaluate healthcare interventions: explanation and elaboration. BMJ 2009; 339: b2700.

22. Moher D, Liberati A, Tetzlaff J, Altman D. Preferred reporting items for systematic reviews and meta-analyses: the PRISMA statement. BMJ 2009; 339: b2535.

23. Page MJ, Shamseer L, Tricco AC. Registration of systematic reviews in PROSPERO: 30,000 records and counting. Syst Rev 2018; 7: 32

24. Bradnova O, Kyrou I, Hainer V, et al. Laparoscopic greater curvature plication in morbidly obese women with type 2 diabetes: effects on glucose homeostasis, postprandial triglyceridemia and selected gut hormones. Obes Surg 2014; 24: 718-26.

25. Ospanov O, Maleckas A, Orekeshova A. Gastric greater curvature plication combined with Nissen fundoplication in the treatment of gastroesophageal reflux disease and obesity. Medicina 2016; 52: 283-90.

26. Doležalova-Kormanova K, Buchwald JN, Skochova D, et al. Fiveyear outcomes: laparoscopic greater curvature plication for treatment of morbid obesity. Obes Surg 2017; 27: 2818-28.

27. Talebpour M, Talebpour A, Barzin G, et al. Effects of laparoscopic gastric plication (LGP) in patients with type 2 diabetes, one year follow-up. J Diabetes Metab Disord 2015; 14: 60.

28. Abdelbaki TN, Huang CK, Ramos A, et al. Gastric plication for morbid obesity: a systematic review. Obes Surg 2012; 22: 1633-9.
29. Buzga M, Maresova P, Seidlerova A, et al. The influence of methods of bariatric surgery for treatment of type 2 diabetes mellitus. Ther Clin Risk Manag 2016; 12: 599-605.

30. Vrbikova J, Kunesova M, Kyrou I, et al. Insulin sensitivity and secretion in obese type 2 diabetic women after various bariatric operations. Obes Facts 2016; 9: 410-23.

31. Sierżantowicz R, Ładny J, Lewko J, Hady H. Assessment of education effects on patient involvement and bariatric treatment outcome: an observational study. Videosurgery Miniinv 2020; 15: 157-65.

32. Nijamkin MP, Campa A, Sosa J, et al. Comprehensive nutrition and lifestyle education improves weight loss and physical actyvity in Hispanic Americans following gastric bypass surgery: a randomized controlled trial. J Acad Nutr Diet 2012; 12: 382-90.

33. Groller KD. Systematic review of patient education practices in weight loss surgery. Surg Obes Relat Dis 2017; 13: 1072-85.

Received: 27.04.2020, accepted: 31.05.2020. 